\title{
Historical Risk Factors Associated with Seizure Outcome After Surgery for Drug-Resistant Mesial Temporal Lobe Epilepsy.
}

\author{
Ali Akbar Asadi-Pooya \\ Maromi Nei \\ Ashwini Sharan \\ Michael R. Sperling
}

Follow this and additional works at: https://jdc.jefferson.edu/neurologyfp

\section{Let us know how access to this document benefits you}

\section{Recommended Citation}

Asadi-Pooya, Ali Akbar; Nei, Maromi; Sharan, Ashwini; and Sperling, Michael R., "Historical Risk Factors Associated with Seizure Outcome After Surgery for Drug-Resistant Mesial Temporal Lobe Epilepsy." (2016). Department of Neurology Faculty Papers. Paper 105.

https://jdc.jefferson.edu/neurologyfp/105

This Article is brought to you for free and open access by the Jefferson Digital Commons. The Jefferson Digital Commons is a service of Thomas Jefferson University's Center for Teaching and Learning (CTL). The Commons is a showcase for Jefferson books and journals, peer-reviewed scholarly publications, unique historical collections from the University archives, and teaching tools. The Jefferson Digital Commons allows researchers and interested readers anywhere in the world to learn about and keep up to date with Jefferson scholarship. This article has been accepted for inclusion in Department of Neurology Faculty Papers by an authorized administrator of the Jefferson Digital Commons. For more information, please contact: JeffersonDigitalCommons@jefferson.edu. 
Title: Historical risk factors associated with seizure outcome after surgery for drug-resistant mesial temporal lobe epilepsy.

\author{
Ali A. Asadi-Pooya, M.D., Maromi Nei , M.D. Ashwini Sharan, M.D., Michael R. Sperling, \\ M.D. \\ Jefferson Comprehensive Epilepsy Center, Department of Neurology, Thomas Jefferson \\ University, Philadelphia, Pennsylvania, U.S.A.
}

\title{
Address for correspondence:
}

Ali A. Asadi-Pooya, M.D.

Department of Neurology

901 Walnut Street, Suite 435

Philadelphia, PA 19107

Phone: 215-955-1222

Fax: 215-955-3745

E-mail: aliasadipooya@yahoo.com; maromi.nei@jefferson.edu; Ashwini.Sharan@jefferson.edu; michael.sperling@jefferson.edu

Running title: Outcome after epilepsy surgery.

Key words: Outcome; Surgery; Temporal lobe epilepsy.

Number of text pages: 13; number of words: 3077; number of words in the abstract: 200; number of references: 29; number of figures: 1 ; number of tables: 1 . 


\begin{abstract}
Objective: To investigate the possible influence of risk factors on seizure outcome after surgery for drug-resistant temporal lobe epilepsy (TLE) and mesial temporal sclerosis (MTS).

Methods: This retrospective study recruited patients with drug-resistant MTS-TLE who underwent epilepsy surgery at Jefferson Comprehensive Epilepsy Center and were followed for a minimum of one year. Patients had been prospectively registered in a database from 1986 through 2014. Post-surgical outcome was classified into two groups; seizure free or relapsed. The possible risk factors influencing long-term outcome after surgery were investigated. Results: 275 patients with MTS-TLE were studied. Two-thirds of the patients had Engel's class 1 outcome and $48.4 \%$ of the patients had sustained seizure freedom, with no seizures since surgery. Patients with a history of tonic-clonic seizures in the year preceding surgery were more likely to experience seizure recurrence (odds ratio $2.4 ; 95 \% \mathrm{CI}: 1.19-4.80 ; \mathrm{p}=0.01$ ). Gender, race, family history of epilepsy, history of febrile seizure, history of status epilepticus, duration of disease before surgery, intelligence quotient, and seizure frequency were not predictors of outcome.
\end{abstract}

Conclusions: Many patients with drug-resistant MTS-TLE respond favorably to surgery. It is critical to distinguish among different types and etiologies of TLE when predicting outcome after surgery. 


\section{Introduction}

Focal epilepsies account for about two-thirds of all adult patients with epilepsy, and temporal lobe epilepsy (TLE) is the most common type of focal epilepsy ${ }^{1,2}$. Mesial temporal sclerosis (MTS) is the most common pathological substrate of $\mathrm{TLE}^{3}$ and about $70 \%$ of patients with drug-resistant TLE show signs of MTS on their MRI ${ }^{4}$. Temporal lobe epilepsy is the most common type of drug-resistant epilepsy referred for epilepsy surgery; it often responds favorably to surgery 5,6 . The potential efficacy of resective surgery in patients with TLE uncontrolled by anti-epileptic drugs is undisputed. However, there are still uncertainties about which patients will benefit most ${ }^{7}$. Various centers use different methodologies in their research. In addition, they often report the combined results from patients with TLE due to MTS and other etiologies as well as nonlesional patients. Such discrepancies may explain the wide variation in seizure free rates after surgery for TLE and they may also obfuscate factors that influence postoperative seizure outcome in these patients ${ }^{6,7}$.

In order to obtain a clearer picture of the possible influence of risk factors on postoperative seizure outcome for patients with drug-resistant MTS-TLE, we conducted a retrospective study of a large surgical cohort. We examined seizure outcome during long-term postsurgical follow-up in patients with magnetic resonance imaging (MRI)-proven MTS-TLE who had undergone epilepsy surgery for drug-resistant seizures. This may shed light on the significance of distinguishing specific types and etiologies of TLE when predicting outcome after surgery. 


\section{Material and methods}

In this retrospective study, we examined data from all patients with a clinical diagnosis of drug-resistant MTS-TLE who underwent epilepsy surgery at Jefferson Comprehensive Epilepsy Center between 1986 and 2014 and had a minimum of 1-year postoperative follow-up. Historical data was obtained by board certified neurologists. The diagnosis of TLE was made by the epileptologists working at this institution and based on clinical grounds (history and semiology) and electroencephalographic (EEG) findings, in order to retain the diagnosis of mesial TLE. There was no age limit to enter the study. All patients underwent a comprehensive presurgical evaluation including a brain MRI, prolonged video-EEG monitoring, neuropsychological evaluation, etc. Magnetic resonance imaging studies were analyzed by neuroradiologists, neurologists, and neurosurgeons with expertise in epilepsy in order to ascertain the diagnosis of MTS. A positive MRI scan for evidence of MTS was required for inclusion in the study. We classified patients as having MTS if they had clear signs of mesial temporal atrophy and/or sclerosis in their brain MRI. Pathology specimens were reviewed by pathologists, but most results were not sufficient to make a precise pathological classification. Patients with dual pathology (in their MRI) and also patients with incomplete data with regard to pre- and postoperative seizure information (i.e., type and frequency) were excluded from this study.

All patients included in the analysis had an anterior temporal lobectomy (ATL).

Resections have evolved over the years, but there has been no difference in treatment strategy. Early on, we performed lateral resections in the dominant hemisphere $4 \mathrm{~cm}$ from the pole; more recently these resections were performed $2-3 \mathrm{~cm}$ from the pole. In the nondominant hemisphere, early on we performed lateral resections $5 \mathrm{~cm}$ from the pole; more recently $3-4 \mathrm{~cm}$ from the pole. In mesial resections there was no difference in resection size between dominant and 
nondominant foci. The patients were followed for at least one year and up to five years after surgery (for many patients the data were missing beyond five years of follow-up). Seizure outcome was monitored periodically by office visits (by the treating physician), telephone contacts, and letters (by the nurse practitioners). Postsurgical outcome was classified into two groups; with sustained seizure freedom or relapsed (one or more seizures after surgery) for the purpose of time to event analysis (Kaplan-Meier estimate of seizure recurrence) and Cox-Mantel test. Aura was not considered as a relapse; only postoperative tonic-clonic seizures and complex partial seizures were considered as relapse. We also used Engel's scale to rate the postoperative seizure outcome.

Age, gender, race, epilepsy risk factors [e.g., history of febrile seizures in childhood, any family history of epilepsy (i.e., first, second, or third degree), etc.], age at seizure onset (i.e., the first afebrile habitual seizure), seizure type(s) and history, date of surgery, date of the first relapse (if any) and date of the last contact with all patients were registered routinely. The type and number of febrile seizures was not registered, so only their presence or absence based on history could be obtained. A history of febrile seizures is registered base on history and details are not only often unavailable, but would be unreliable because of the retrospective nature of these reports.

Demographic variables and relevant clinical variables were summarized descriptively to characterize the study population. Potential outcome predictors, including gender, age at surgery, duration of epilepsy, race, history of status epilepticus before surgery, history of tonic-clonic seizures before surgery, history of febrile seizures in childhood, family history of epilepsy, full scale intelligence quotient (IQ), and preoperative seizure frequency were assessed comparing seizure free and non-seizure free patients, who happened to have similar duration of 
postoperative follow-up ( $4.1 \pm 1.3$ vs. $4.4 \pm 1.1$ years, respectively). Initially, we performed univariate analyses using Pearson Chi-square, Mann-Whitney, Kolmogorov-Smirnov, and t-test. Variables that were significant or nearly so in univariate tests were assessed in a logistic regression. Time to event analysis was used to produce a Kaplan-Meier estimate of seizure recurrence. A Kaplan-Meier analysis allows estimation of event over time, even when patients drop out or are studied for different lengths of time. Cox-Mantel test was used to study annual rate of occurrence of first seizure after resective epilepsy surgery in different groups of patients. Odds ratio and $95 \%$ confidence interval (CI) were calculated. P value less than 0.05 was considered as significant. No informed consent was required, as it was a retrospective study. This study was conducted with the approval by Thomas Jefferson University Institutional Review Board. 


\section{Results}

From 1986 until 2014, 789 patients had anterior temporal lobectomy at our center; 770 patients had at least one year of follow-up. Of these, 309 patients had MTS in their MRI, 251 patients had other abnormalities (e.g., tumor, gliosis, vascular lesion, etc.) in their MRIs, 168 patients had a normal MRI, 19 patients had dual pathology and MRI was not available in 23 patients. Among patients with MTS, 34 patients had incomplete data; 275 patients (132 males and 143 females) had MTS-TLE with at least one year of postoperative follow-up and complete seizure data; these were studied. One hundred thirty-three (48.4\%) patients had sustained seizure freedom and $142(51.6 \%)$ patients experienced one or more seizures after surgery. When we classified postoperative seizure outcome according to Engel's scale, 259 patients had available data; of these, 172 patients $(66.4 \%)$ were seizure free (Engel's class 1 outcome). For the statistical purposes, we considered 133 patients as seizure free (those with sustained seizure freedom; see above), as in this long-term postsurgical follow-up study some patients dropped out or were studied for different lengths of time. Univariate analyses of risk factors for recurrence of postoperative seizures appear in Table 1 . The analysis revealed an association between a history of preoperative tonic-clonic seizures and postoperative seizure recurrence. Preoperative seizure frequency was also associated with seizure outcome. Subsequent logistic regression analysis to assess these variables showed that a history of preoperative tonic-clonic seizures in the year preceding surgery was a strong predictor, and the only predictor, of postoperative seizure recurrence (Figure 1). Frequency of complex partial seizures $(\mathrm{p}=0.2)$ or tonic-clonic seizures $(\mathrm{p}$ $=0.1$ ) was not a significant risk factor. Patients who had recent preoperative tonic-clonic seizures (i.e., in the year preceding surgery) had a greater chance of seizure recurrence than those without such a history (odds ratio 2.4; 95\% CI: 1.19-4.80; $\mathrm{p}=0.01$ ). The model correctly predicted 
$58.5 \%$ of the cases of relapse. However, a remote history of preoperative tonic-clonic seizures was not a risk factor for seizure recurrence. Among patients who had a remote history of preoperative tonic-clonic seizures (i.e., not within the year preceding their surgery), chance of having postoperative seizure recurrence was similar to those who did not have any preoperative tonic-clonic seizures (odds ratio: $1.31,95 \% \mathrm{CI}: 0.74-2.31 ; \mathrm{p}=0.3$ ). Total preoperative seizure frequency (complex partial seizures plus tonic-clonic seizures) was calculated and patients were dichotomized into those with less than 20 seizures per month versus patients with 20 or more seizures per month; this was not related to outcome $(\mathrm{p}=0.9)$. In addition, epilepsy duration (less than 20 years compared to more than 20 years of epilepsy) did not influence outcome $(\mathrm{p}=0.5)$.

In a subanalysis, we studied patients who were seizure free and aura free for one year or more in their last follow-up visit (77 patients) compared with those who had any seizures or auras (198 patients); this subanalysis did not affect the results. A history of preoperative tonicclonic seizures $(p=0.005)$ and also preoperative tonic-clonic seizure frequency $(p=0.002)$ were associated with outcome in univariate analyses. We then performed a logistic regression analysis, assessing these variables. A history of preoperative tonic-clonic seizures in the year preceding surgery showed a trend in predicting postoperative seizure recurrence. Recent preoperative tonicclonic seizures (i.e., in the year preceding surgery) were associated with a greater chance of seizure recurrence (odds ratio $2.15 ; 95 \%$ CI: $0.96-4.82 ; \mathrm{p}=0.06$ ). The model correctly predicted $71.9 \%$ of the cases of relapse. 


\section{Discussion}

Drug-resistant temporal lobe epilepsy is a relatively common type of epilepsy that often responds favorably to surgery. In our study, about half of the patients had sustained seizure freedom during a long-term postsurgical follow-up, which is concordant with most previous studies $6,7,10,11$. This study aimed to resolve ambiguities regarding the prognostic value of historical risk factors in drug-resistant TLE after surgery in patients with MTS. We found that a history of recent preoperative tonic-clonic seizures was associated with postoperative seizure recurrence.

\section{History of febrile seizures in childhood}

A history of febrile seizure in the past did not predict the postoperative outcome in patients with drug-resistant MTS-TLE. A previous meta-analysis of predictors of temporal lobectomy outcome in a heterogeneous population of TLE patients found febrile seizures to be a strong prognostic indicator of seizure remission (odds ratio $0.48 ; 95 \%$ confidence interval $0.27-0.83)^{7}$. However, other studies that only included patients with MTS found that a history of febrile seizure in childhood did not predict the seizure outcome after surgery ${ }^{12,13}$; our study findings are concordant with these studies. Febrile seizures are strongly associated with MTS, which itself is associated with a favorable prognosis. However, as an independent predictor, febrile seizure only predicts outcome when MTS is one of many underlying pathologies in patients with diverse etiologies for their epilepsy. It loses its predictive value when the subgroup of patients with MTS is examined ${ }^{13}$. Febrile seizures are clearly related to MTS at times ${ }^{14}$. MRI findings of a markedly hyperintense hippocampus in children with febrile status epilepticus was highly 
associated with later development of mesial temporal sclerosis ${ }^{14,15}$. It appears that it is critical to distinguish among different types and etiologies of TLE when predicting outcome after surgery.

\section{History of tonic-clonic seizures}

In our study, the only factor predictive of seizure outcome after surgery in patients with drug-resistant MTS-TLE was the occurrence of tonic-clonic seizures, if there were any in the year preceding surgery. When MRI or pathology clearly shows MTS, then preoperative tonicclonic seizures reduce the chance of postoperative seizure freedom ${ }^{12}$. Another study with a more heterogeneous population of 280 patients who underwent anterior temporal lobectomy also associated a history of secondary generalized tonic-clonic seizure(s) with a lower probability of becoming seizure free (odds ratio $0.47,95 \%$ CI $0.25-0.90 ; p=0.02$ ) ${ }^{16}$. Another study of 339 patients followed for more than 2 years found that absence of generalized tonic-clonic seizures and presence of hippocampal atrophy on MRI were significantly associated with remission in patients with medial temporal resection ${ }^{17}$. Recent tonic-clonic seizures in a patient with MTS may reflect more widespread epileptogenicity extending beyond the borders of mesial temporal structures. Evidence obtained with fMRI suggests significant, more widespread derangements in brain function and connectivity in TLE patients with tonic-clonic seizures (compared with those without this seizure type) ${ }^{18}$.

\section{Duration of epilepsy before surgery}

Duration of epilepsy before surgery is determined by age at the onset of epilepsy and age at surgery. In our study, duration of epilepsy did not predict seizure outcome after epilepsy surgery in patients with drug-resistant MTS-TLE. Another study of 116 consecutive patients who 
had temporal lobe surgery for drug-resistant epilepsy and MTS reported similar observations to ours ${ }^{12}$. In contrast, another retrospective study which included 434 consecutive adult patients who underwent TLE surgery found that longer epilepsy duration correlated with poor outcome ${ }^{9}$. These authors investigated a heterogeneous group of patients including those with MTS and others with tumors or focal cortical dysplasia. As in other variables, better definition of the location within the temporal lobe of the epileptogenic zone may provide better insights.

\section{Intelligence quotient (IQ)}

In our study, IQ did not predict postoperative seizure outcome in patients with MTSTLE. In one previous study, the authors examined the relationship between baseline IQ scores and seizure outcome in 1,034 temporal lobectomy cases from eight epilepsy surgery centers ${ }^{19}$. Those patients who continued to have seizures following surgery had statistically lower preoperative IQ scores than those who were seizure free $(\mathrm{p}<0.009)$, but only by 2.3 points. There was considerable overlap between the two groups. However, relative risk analyses revealed no significant increase in risk of surgical failure among patients with low IQ scores who had no structural lesions other than mesial temporal sclerosis ${ }^{19}$. This is consistent with our findings. However, another study that included all resective epilepsy surgeries found a significant relationship between IQ and seizure freedom, with a higher IQ associated with a better outcome ${ }^{20}$. Intelligence quotient may be relevant in epilepsy surgery when etiology is not considered, but appears to be of no importance in the subset of patients with MTS-TLE.

\section{Seizure frequency}


In our study, frequency of preoperative habitual seizures (i.e., either complex partial or generalized tonic-clonic seizures) was not associated with postoperative seizure outcome in patients with MTS-TLE. The data with regard to the role of seizure frequency is inconsistent in the literature. In a previous study of 79 patients with TLE (all etiologies) ${ }^{8}$, those with more than 20 seizures per month were significantly less likely to be seizure free after temporal lobectomy than the rest of patients $(\mathrm{p}=0.009)$. However, another study found that in patients who had anterior temporal lobectomy, preoperative seizure frequency was not associated with postoperative seizure frequency, while in non-ATL operations, preoperative seizure frequency of $\geq 20$ seizures per month was associated with frequent postoperative seizures $(p=0.03)^{21}$. Our data suggests that the frequency of preoperative habitual seizures does not affect surgery outcome in patients with drug-resistant MTS-TLE.

\section{Status epilepticus}

In our study, a history of status epilepticus in the past did not predict postoperative outcome in patients with MTS-TLE. This is similar to a previous study of 116 consecutive patients who had temporal lobe surgery for drug-resistant epilepsy and MTS ${ }^{12}$, and contrasts with the findings of another previous study of 118 patients with MTS ${ }^{13}$. A large multicenter study would help assess this issue.

\section{Gender}

In our study, gender did not predict postoperative outcome in patients with MTS-TLE. One previous study of 252 patients, who underwent surgical treatment for mesial temporal sclerosis, showed that male sex was associated with a reduced risk of seizure recurrence (odds ratio, 0.5; 
$95 \%$ confidence interval, $0.3-0.8)^{22}$. However, the same authors in a more recent paper including a larger sample size (368 patients) concluded that gender is not a predictor of long-term outcome following surgery for temporal lobe epilepsy ${ }^{23}$. Other studies ${ }^{13}$ have shown similar results to ours.

\section{Race}

Race was not a predictor of surgery outcome in patients with drug-resistant MTS-TLE, in our study. The vast majority of previous studies ${ }^{23}$ have the same observation. However, one previous study showed a trend for higher rates of seizure recurrence in African-Americans compared with white patients ${ }^{24}$. That study was limited by the small sample size (i.e., 64 patients). In general, epidemiologically race does not seem to affect postoperative seizure outcome in patients with drug-resistant MTS-TLE.

\section{Family history of epilepsy}

A family history of epilepsy did not predict postoperative outcome in patients with MTSTLE, in our study. Similar results were reported in a previous study of 118 patients with MTS ${ }^{13}$. However, another retrospective study of 434 consecutive patients, who underwent TLE surgery, found a family history of epilepsy to be a predictor of remission ${ }^{9}$. In the latter study, the authors investigated a heterogeneous group of patients including those with MTS, tumors or focal cortical dysplasia. As said before, better definition of the location of the epileptogenic zone may provide better insights. 


\section{Conclusions}

It is well known that there are several subgroups in TLE (e.g., MTS, TLE plus, pseudoTLE, etc.) ${ }^{25,26}$. This research provides further support that differentiating TLE subtypes may ultimately lead to a better understanding of the pathophysiology of the temporal lobe epilepsies. The potential efficacy of resective surgery in patients with TLE uncontrolled by anti-epileptic drugs is undisputed ${ }^{27-29}$. However, it is critical to distinguish among different types and etiologies of TLE when predicting seizure outcome after surgery.

Our study places historical risk factors into the equation; however, they are not the only predictors of postoperative seizure outcome. It is essential that all factors (e.g., EEG, other imaging studies, neuropsychological profile, surgery techniques and volume of resection, etc.) are taken into account. In order to improve algorithms for predicting postoperative seizure outcome in patients with drug-resistant epilepsy, our findings should be combined with those from other studies.

\section{Study limitations}

This was a retrospective study and patient data came from a single institution. Imaging techniques have changed with equipment modernization and the general ability to detect abnormalities on MRI scans has improved. Other non-historical factors (e.g., resection size) are not taken into account in this study. Additionally, resections have evolved somewhat over the

years, but there has been no difference in treatment strategy. Finally, pathology specimens were reviewed by pathologists, but most results were not sufficient to provide a precise pathological classification. 


\section{Conflict of interest}

Ali A. Asadi-Pooya, M.D., reports no disclosures.

Maromi Nei, M.D., Research: Upsher-Smith Laboratories, NINDS

Ashwini Sharan, M.D., Consulting, Clinical Trial Site - Medtronic; SJM, Clinical Trial Site - Grant, honorarium; ICVRX - ownership interest; ICP - ownership interest; Tiger labs ownership interest; Saluda Medical - Clinical Trial Site.

Michael R. Sperling, M.D., Consulting: UCB Pharma; Research: contracts with Thomas Jefferson University, Eisai, UCB Pharma, Sunovion, SK Life Sciences, Marinus, Lundbeck, Medtronics, Visualase, Accorda, Upsher-Smith, Brain Sentinel.

\section{Acknowledgment}

This was a non-funded study. We thank Jennifer Fisher Wilson for editorial assistance. 


\section{Reference}

1. Téllez-Zenteno JF, Hernández-Ronquillo L. A review of the epidemiology of temporal lobe epilepsy. Epilepsy Res Treat 2012; 2012: 630853.

2. Semah F, Picot MC, Adam C, et al. Is the underlying cause of epilepsy a major prognostic factor for recurrence? Neurology 1998; 51(5): 1256-1262.

3. Wiebe S. Epidemiology of temporal lobe epilepsy. Can J Neurol Sci 2000; 27 Suppl 1: S6-10.

4. Wiebe S, Blume WT, Girvin JP, Eliasziw M. Effectiveness, Efficiency of Surgery for Temporal Lobe Epilepsy Study G. A randomized, controlled trial of surgery for temporallobe epilepsy. N Engl J Med 2001; 345: 311-318.

5. Kumlien E, Doss RC, Gates JR. Treatment outcome in patients with mesial temporal sclerosis. Seizure 2002; 11(7): 413-417.

6. Fong JS, Jehi L, Najm I, Prayson RA, Busch R, Bingaman W. Seizure outcome and its predictors after temporal lobe epilepsy surgery in patients with normal MRI. Epilepsia 2011; 52(8): 1393-1401.

7. Tonini C, Beghi E, Berg AT, et al. Predictors of epilepsy surgery outcome: a metaanalysis. Epilepsy Res 2004; 62(1): 75-87.

8. Foldvary N, Nashold B, Mascha E, et al. Seizure outcome after temporal lobectomy for temporal lobe epilepsy: a Kaplan-Meier survival analysis. Neurology 2000; 54(3): 630634.

9. Elsharkawy AE, Alabbasi AH, Pannek H, et al. Long-term outcome after temporal lobe epilepsy surgery in 434 consecutive adult patients. J Neurosurg 2009; 110(6): 1135-1146. 
10. Cukiert A, Burattini JA, Mariani PP, et al. Outcome after cortico-amygdalohippocampectomy in patients with temporal lobe epilepsy and normal MRI. Seizure 2010; 19(6): 319-323.

11. Yang PF, Pei JS, Zhang HJ, et al. Long-term epilepsy surgery outcomes in patients with PET-positive, MRI-negative temporal lobe epilepsy. Epilepsy Behav 2014; 41: 91-97.

12. Hennessy MJ, Elwes RD, Rabe-Hesketh S, Binnie CD, Polkey CE. Prognostic factors in the surgical treatment of medically intractable epilepsy associated with mesial temporal sclerosis. Acta Neurol Scand 2001; 103(6): 344-350.

13. Hardy SG, Miller JW, Holmes MD, et al. Factors predicting outcome of surgery for intractable epilepsy with pathologically verified mesial temporal sclerosis. Epilepsia 2003; 44(4): 565-568.

14. Lewis DV, Shinnar S, Hesdorffer DC, et al; FEBSTAT Study Team. Hippocampal sclerosis after febrile status epilepticus: the FEBSTAT study. Ann Neurol 2014; 75(2): 178-185.

15. Provenzale JM, Barboriak DP, Van Landingham K, MacFall J, Delong D, Lewis DV. Hippocampal MRI signal hyperintensity after febrile status epilepticus is predictive of subsequent mesial temporal sclerosis. Am J Roentgenol 2008; 190(4): 976-983.

16. Cleary RA, Thompson PJ, Fox Z, Foong J. Predictors of psychiatric and seizure outcome following temporal lobe epilepsy surgery. Epilepsia 2012; 53(10): 1705-1712.

17. Spencer SS, Berg AT, Vickrey BG, et al; Multicenter Study of Epilepsy Surgery. Predicting long-term seizure outcome after resective epilepsy surgery: the multicenter study. Neurology 2005; 65(6): 912-918. 
18. He X, Doucet G, Sperling MR, Sharan A, Tracy J. Reduced thalamocortical functional connectivity in temporal lobe epilepsy. Epilepsia 2015; in press.

19. Chelune GJ, Naugle RI, Hermann BP, et al. Does presurgical IQ predict seizure outcome after temporal lobectomy? Evidence from the Bozeman Epilepsy Consortium. Epilepsia 1998; 39(3): 314-318.

20. Malmgren K, Olsson I, Engman E, Flink R, Rydenhag B. Seizure outcome after resective epilepsy surgery in patients with low IQ. Brain 2008; 131(Pt 2): 535-542.

21. Khoury JS, Winokur RS, Tracy JI, Sperling MR. Predicting seizure frequency after epilepsy surgery. Epilepsy Res 2005; 67(3): 89-99.

22. Burneo JG, Black L, Martin R, et al. Race/ethnicity, sex, and socioeconomic status as predictors of outcome after surgery for temporal lobe epilepsy. Arch Neurol 2006; 63(8): 1106-1110.

23. Burneo JG, Villanueva V, Knowlton RC, Faught RE, Kuzniecky RI. Kaplan-Meier analysis on seizure outcome after epilepsy surgery: do gender and race influence it? Seizure 2008; 17(4): 314-319.

24. Burneo JG, Knowlton RC, Martin R, Faught RE, Kuzniecky RI. Race/ethnicity: a predictor of temporal lobe epilepsy surgery outcome? Epilepsy Behav 2005; 7: 486-490.

25. Kahane P, Bartolomei F. Temporal lobe epilepsy and hippocampal sclerosis: lessons from depth EEG recordings. Epilepsia 2010; 51 Suppl 1: 59-62.

26. Barba C, Barbati G, Minotti L, Hoffmann D, Kahane P. Ictal clinical and scalp-EEG findings differentiating temporal lobe epilepsies from temporal 'plus' epilepsies. Brain 2007; 130(Pt 7): 1957-1967. 
27. Sperling MR, O'Connor MJ, Saykin AJ, Plummer C. Temporal lobectomy for refractory epilepsy. JAMA 1996; 276(6): 470-475.

28. Téllez-Zenteno JF, Hernández Ronquillo L, Moien-Afshari F, Wiebe S. Surgical outcomes in lesional and non-lesional epilepsy: a systematic review and meta-analysis. Epilepsy Res 2010; 89(2-3): 310-318.

29. Schmidt D, Stavem K. Long-term seizure outcome of surgery versus no surgery for drugresistant partial epilepsy: a review of controlled studies. Epilepsia 2009; 50(6): 13011309. 